Available online at GSC Online Press Directory

GSC Biological and Pharmaceutical Sciences

e-ISSN: 2581-3250, CODEN (USA): GBPSC2

Journal homepage: https://www.gsconlinepress.com/journals/gscbps

(RESEARCH ARTICLE)

\title{
Monosodium glutamate alter hepatic functions, redox potential and lipid metabolism: Omega 3 fatty acids ameliorative intervention
}

\author{
Divine Avwerosuoghene Onobrudu ${ }^{1,}{ }^{*}$ and Barine Innocent Nwiloh ${ }^{2}$ \\ ${ }^{1}$ Medical Biochemistry Department, Delta State University, Abraka, Delta State, Nigeria. \\ ${ }^{2}$ Natural Science Unit, School of General Studies, University of Nigeria, Nsukka, Enugu State, Nigeria.
}

Publication history: Received on 02 October 2020; revised on 12 October 2020; accepted on 16 October 2020

Article DOI: https://doi.org/10.30574/gscbps.2020.13.1.0324

\begin{abstract}
Monosodium glutamate (MSG) toxicity is fast becoming a global health challenge due to the increase in its consumption as a food additive. This study investigated the effect of consumption of MSG and treatment with graded doses of omega 3 fatty acids ( $\omega-3)$. Forty-eight male Wistar rats $(n=8)$ grouped into six; control, MSG, MSG + Low dose of $\omega-3$ (LD $\omega-3$ ); MSG + High dose of $\omega-3$ (HD $\omega-3$ ), LD $\omega-3$, and HD $\omega-3$ were used for this study. MSG was administered at $4 \mathrm{~g} / \mathrm{L} / \mathrm{day}$ in their drinking water for 6 weeks, while $\omega-3$ was administered at low and high doses of 100 and $300 \mathrm{mg} / \mathrm{kg} \mathrm{BW,} \mathrm{p.0.}$ respectively for 4 weeks. Results revealed that administration of MSG induced imbalance in lipid metabolism, oxidative stress and hepatic dysfunction. These were revealed by significant decreases in TG, HDL-C, CAT, GSH, albumin and total protein; but, significant increases in LDL-C, MDA, AST, ALT, ALP, and total bilirubin (TB), compared to control group. Administration of graded doses of $\omega-3$ following treatment with MSG was characterized with significant reductions in ALT, ALP, TB and MDA. The administration of $\omega-3$ showed no effects on the antioxidant indices. Conclusively, LD $\omega-3$ is a potent ameliorative supplement which can be administered after pre-exposure to MSG.
\end{abstract}

Keywords: Food additives; Hepatotoxicity; Monosodium glutamate; Omega 3 fatty acids, Dyslipidemia

\section{Introduction}

Monosodium glutamate (MSG) is an amino acid, the sodium salt of glutamic acid. It is a white crystalline salt that produces a palatable taste referred to as "umami" [1,2]. MSG was first identified by Kikunae Ikeda as a taste enhancer in 1908 at Tokyo Imperial University where it was extracted from Lamineria japonica a sea weed [1]. At present it is commonly utilize in the production of most food seasonings to enhance taste. Glutamate has been reported to function in some biological processes, such as syntheses of neurotransmitters (gamma-aminobutyric acid, nitric oxide), folic acid, collagen and glutathione [3]. MSG is used by glutamate receptors in nerve terminals, brain, spinal cord, conducting system, kidney, liver, testes, lungs, spleen and heart [4].

The estimated daily consumption of MSG is $0.55 \mathrm{~g} /$ day [5]. However, due to increased consumption of processed foods with MSG, the daily intake in most developed and developing countries could rise as high as 3-4g/day [6]. Regular consumption of high amount of MSG was proven to be harmful in both animal and human trials [4, 7]. At present the safety of MSG consumption is questionable. Previous report has shown that monosodium glutamate have deteriorating effect on some organs and it disrupt cell functions which could lead to several health disorders [8]. It has been implicated in obesity [7], diabetics, hepatotoxicity, neurotoxicity and genotoxicity [8]. MSG toxicity is fast becoming a major cause of concern due to its uprising consumption and demand hence the need to investigate palliative therapies that could obstruct and ameliorate MSG stimulated pathological processes.

\footnotetext{
${ }^{*}$ Corresponding author: Divine Avwerosuoghene Onobrudu

Medical Biochemistry Department, Delta State University, Abraka, Nigeria. suoana2000@yahoo.com
} 
We were excited to investigate Eicosapentanoic acid (EPA) and docosahexaenoic (DHA) acid which are polyunsaturated fatty acids frequently called omega- 3 fatty acids [9], which are commonly found naturally in sea fishes such as cod liver, salmon, Mackerel, herring [10,11]. Presently, there are reports on the roles of EPA and DHA in the management of health challenges, although their mechanism of action is presently not completely understood. Omega 3 fatty acids possess antioxidant properties that supported its use in amelioration and inhibition of oxidative stress [12,13]. The ameliorative role of EPA and DHA in diabetics [14], rheumatoid arthritis and infant development [15], breast cancer [16], aveolar bone loss [17] and cardiovascular disorders and mental health have been proven $[10,18,19,20]$. Thus, the decision to evaluate the pharmacological potentials of omega-3 fatty acids on selected biomarkers in monosodium glutamate induced rats.

\section{Material and methods}

\subsection{Chemicals and Drugs}

Monosodium glutamate (CAS No. 142-47-2; purity: $\geq 99.5 \%$ ) was provided by Sigma - Aldrich, St. Louis, MO, USA. Omega 3 fatty acid was obtained from Emzor pharmaceutical Industries Limited, Lagos, Nigeria. Diagnostic kits for the determination of total cholesterol, triglyceride and high density lipoprotein cholesterol, aspartate aminotransferase, alanine aminotransferase, alkaline phosphatase, total bilirubin, direct bilirubin, albumin, and total protein were supplied by Randox Laboratories Ltd., United kingdom.

\subsection{Experimental Animals}

Forty-eight (48) adult male Wistar rats weighing between 180 and 200 g were acquired from animal house, Delta State University, Abraka, Delta State, Nigeria. They were kept in the Animal House of Chemical Sciences, Novena University, in plastic cages at room temperature and photo-periodicity of about $12 \mathrm{hrs}$ light/12 hrs dark. The rats were given rodent pellet and water ad libitum, and weighed weekly. After seven days of acclimatization, the animals were randomly allotted to separate groups.

\subsection{Experimental Design}

The rats were divided into six groups of 8 rats each and treated as follows: Group 1 (Control), 3 (LD $\omega-3$ ) and 4 (HD $\omega$ 3) received normal saline while group 2 (MSG), 5 (MSG + LD $\omega-3$ ) and 6 (MSG + HD $\omega-3$ ) received $4.0 \mathrm{mg} / \mathrm{kg}$ body weight p.o. of monosodium glutamate. The $4.0 \mathrm{mg} / \mathrm{kg}$ body weight monosodium glutamate was adopted from Lopez-miranda et al., [5] and it corresponds to about $0.45 \mathrm{~g} / \mathrm{kg} /$ day [21]. Fourteen (14) days post administration of monosodium glutamate the treatment with omega 3 fatty acid commenced for a duration of twenty eight (28) days. Omega 3 fatty acids was administered at a low dose of $100 \mathrm{mg} / \mathrm{kg}$ bodyweight, p.o. to groups 3 and 5, and at a high dose of $300 \mathrm{mg} / \mathrm{kg}$ bodyweight p.o. to groups 4 and 6 . At the end of the treatment period the the animals were administered sodium pentobarbital anesthesia at $40 \mathrm{mg} / \mathrm{kg}$, i.m., dissected and the blood collected by cardiac puncture into plain and lithium oxalate sample bottles. Thereafter, the bottles were centrifuged at 1200 revolutions per minute for 10 minutes. The supernatant of each sample were collected into separate labelled plain bottles, and the bioassays were done immediately.

\subsection{Biochemical Analysis}

The levels of reduced glutathione was determined according to the method of Sedlak and Lindsay [22], malondialdehyde was determined according to the method of Hunter et al., [23] as modified by Gutteridge and Wilkins [24], while the activities of catalase and superoxide dismutase were determined by the method of Beers and Sizer [25], and by the method of Mistra and Fridovich [26] respectively

The levels of total cholesterol, triglyceride and high density lipoprotein cholesterol, total bilirubin, direct bilirubin, albumin, total protein and activities of aspartate aminotransferase, alanine aminotransferase and alkaline phosphatase were determined colorimetrically using Lasany Microprocesssor single beam visible Spectrophotometer LI-721 and Randox diagnostic kit. Assays were performed according to the manufacturer's instructions.

\subsection{Data Analysis}

The data obtained from this study were analyzed using statistical package for social sciences version 18.0. Statistical assessments of the differences between the group mean values were tested by one way analysis of variance (ANOVA), and then Turkey post-hoc test for multiple comparisons. The results were presented in graphical and tabular forms using the mean \pm standard deviation (SD). Statistical significance was considered at $p \leq 0.05$. 


\section{Results and discussion}

3.1. Effect of graded doses of omega 3 fatty acids ( $\omega$-3) on total cholesterol (TC), triglyceride (TG), low and high density lipoprotein cholesterol (LDL-C and HDL-C) in monosodium glutamate (MSG) treated male Wistar rats

As shown in figure 1-4, when compared to the control group, there were significant $(p<0.05)$ increase in TC level in LD $\omega-3$ and HD $\omega-3$ groups; in TG level in LD $\omega-3$ and MSG + LD $\omega-3$ groups. However, significant $(p<0.05)$ increases were noted in LDL-C in groups 2-5 (MSG, LD $\omega-3, \mathrm{HD} \omega-3$, and MSG + LD $\omega-3$ respectively), but a significant $(p<0.05)$ elevation was only recorded in HDL-C level in HD $\omega-3$ group. Contrarily, significant $(p<0.05)$ reductions were observed in TG in MSG and HD $\omega-3$ groups and in HDL-C in MSG, LD $\omega-3$ and MSG + LD $\omega-3$ groups respectively.

When compared to MSG group, significant $(p<0.05)$ increases were observed in TC level in HD $\omega-3$ and HD $\omega-3$ groups and in TG in LD $\omega-3$, MSG + LD $\omega-3$ and MSG+HD $\omega$-3 groups; while significant $(p<0.05)$ decreases were noted in LDL$\mathrm{C}$ in groups 4-6 (HD $\omega-3$, and MSG + LD $\omega-3$, MSG+HD $\omega-3$ respectively). However a significant $(p<0.05)$ elevation was only seen in HDL-C in HD $\omega-3$ group.

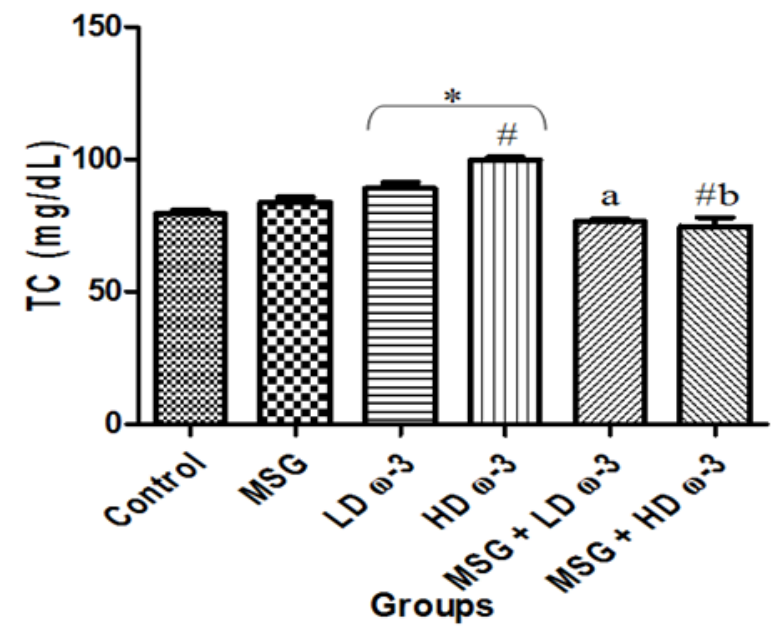

Graph 1. Effect of omega 3 fatty acids ( $\omega$-3) on total cholesterol (TC) in monosodium glutamate (MSG) treated rats.

Values were expressed as mean $\pm \mathrm{SD}$, values with superscript $*$ is significantly different at $p \leq 0.05$ when compared to

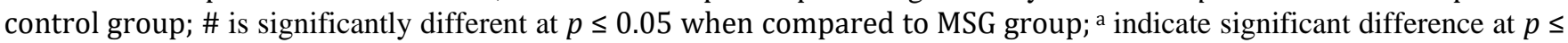
0.05 between LD $\omega-3$ and MSG + LD $\omega-3$; b indicate significant difference at $p \leq 0.05$ between HD $\omega-3$ and MSG + HD $\omega-3$.

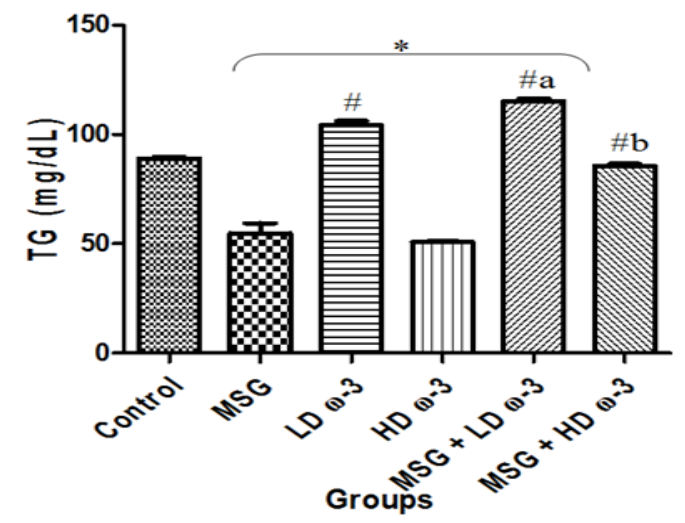

Graph 2. Effect of omega 3 fatty acids ( $\omega$-3) on triglyceride (TG) in monosodium glutamate (MSG) treated Wistar rats. 


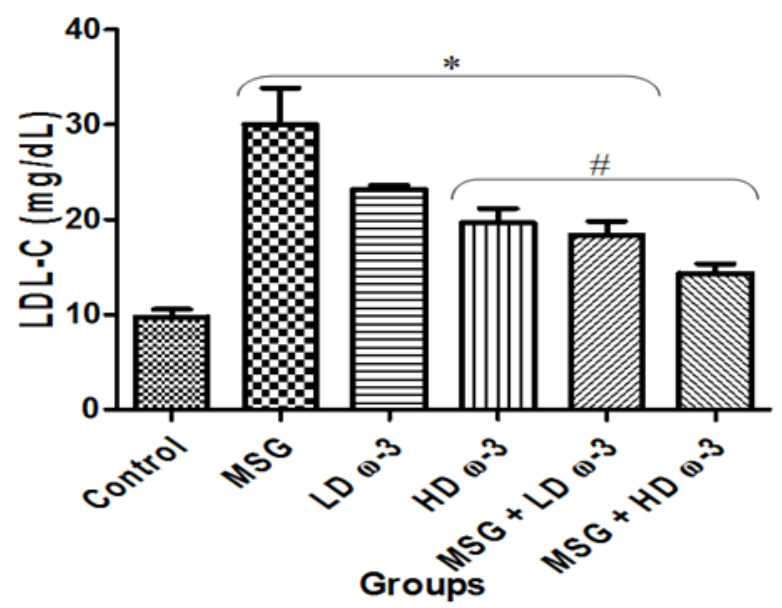

Graph 3. Effect of omega 3 fatty acids ( $\omega$-3) on low density lipoprotein cholesterol (LDL-C) in monosodium glutamate (MSG) treated Wistar rats.

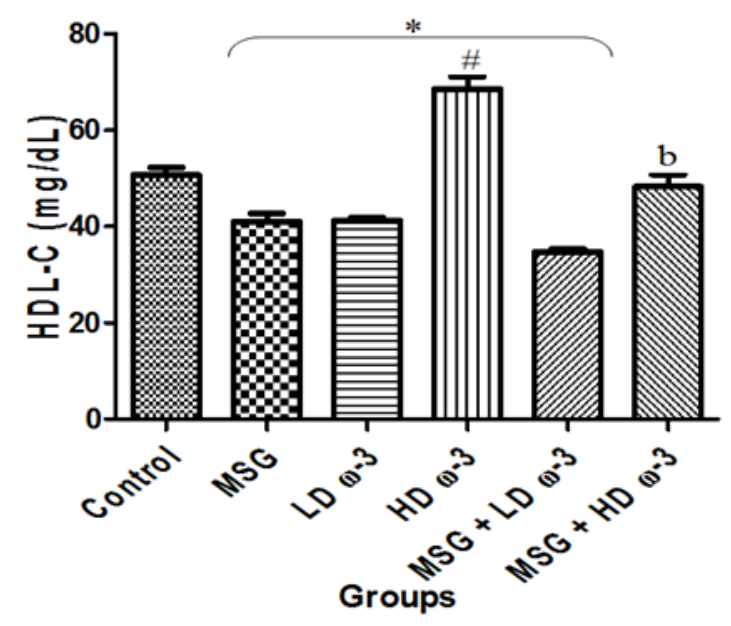

Graph 4. Effect of omega 3 fatty acids ( $\omega-3)$ on high density lipoprotein cholesterol (HDL-C) in monosodium glutamate (MSG) treated Wistar rats.

\subsection{Effect of graded doses of omega 3 fatty acids ( $\omega-3)$ on malondialdehyde (MDA), superoxide dismutase (SOD), catalase (CAT) and glutathione (GSH) in monosodium glutamate (MSG) treated Wistar rats}

As shown in graph 5, when compared to the control group there were significant $(p<0.05)$ increase in MDA level in MSG and MSG + HD $\omega-3$ groups. However, compared to MSG, there were significant $(p<0.05)$ reductions in MDA level in groups 3-5 (LD $\omega-3, \mathrm{HD} \omega-3$ and MSG+LD $\omega-3)$.

Relative to the control group, there were significant $(p<0.05)$ reduction in GSH level and CAT activities in MSG, MSG+LD $\omega-3$ and MSG+ HD $\omega-3$. However, significant $(p<0.05)$ increase was observed in SOD activities in MSG and MSG + LD $\omega-$ 3 groups when compared to control group. Relative to MSG group, there were significant $(p<0.05)$ increase in the activity of CAT in LD $\omega-3$ and HD $\omega-3$ groups, but significant $(p<0.05)$ reduction in SOD activities in LD $\omega-3$, HD $\omega-3$, MSG + LD $\omega-3$ and MSG + HD $\omega-3$ groups. Although there were increases in the level of GSH, but these changes were not significant at $p<0.05$. Relative to LD $\omega-3$ group, there is a significant $(p<0.05)$ increase in the activity of SOD although, there were significant $(p<0.05)$ reduction in MDA, CAT and GSH in MSG + LD $\omega-3$ group. Moreover, relative to HD $\omega-3$ group, there was a significant $(p<0.05)$ elevation in MDA level, and a significant $(p<0.05)$ reduction in GSH activity in MSG + HD $\omega-3$ group (Graph 6-8). 


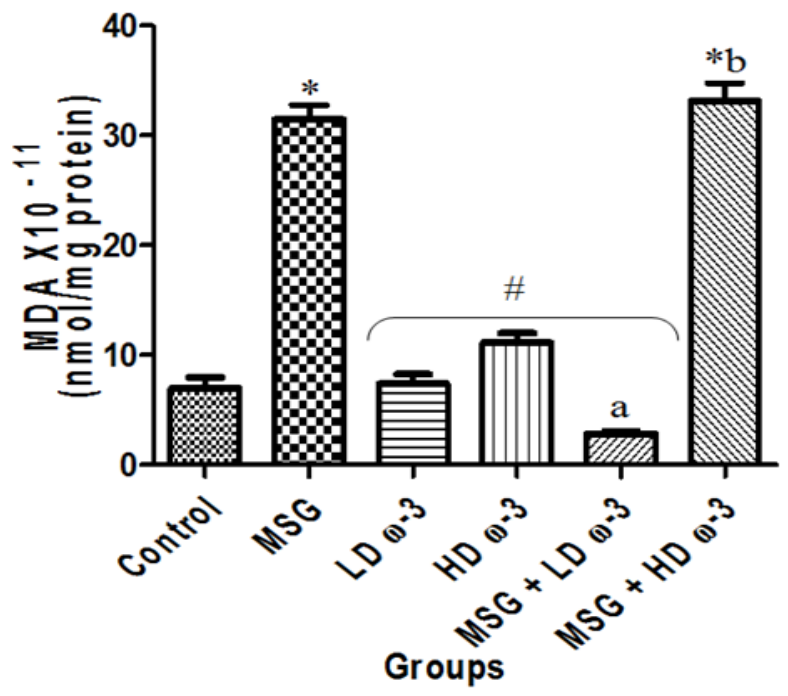

Graph 5. Effect of omega 3 fatty acids ( $\omega-3)$ on malondialdehyde (MDA) in monosodium glutamate (MSG) treated Wistar rats.

Values were expressed as mean $\pm \mathrm{SD}$, values with superscript $*$ is significantly different at $p \leq 0.05$ when compared to

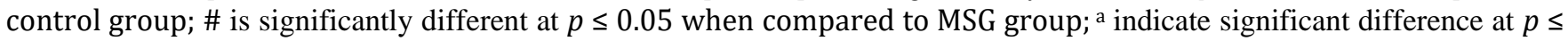
0.05 between LD $\omega-3$ and MSG + LD $\omega-3$; ${ }^{\mathrm{b}}$ indicate significant difference at $p \leq 0.05$ between HD $\omega-3$ and MSG + HD $\omega-3$.

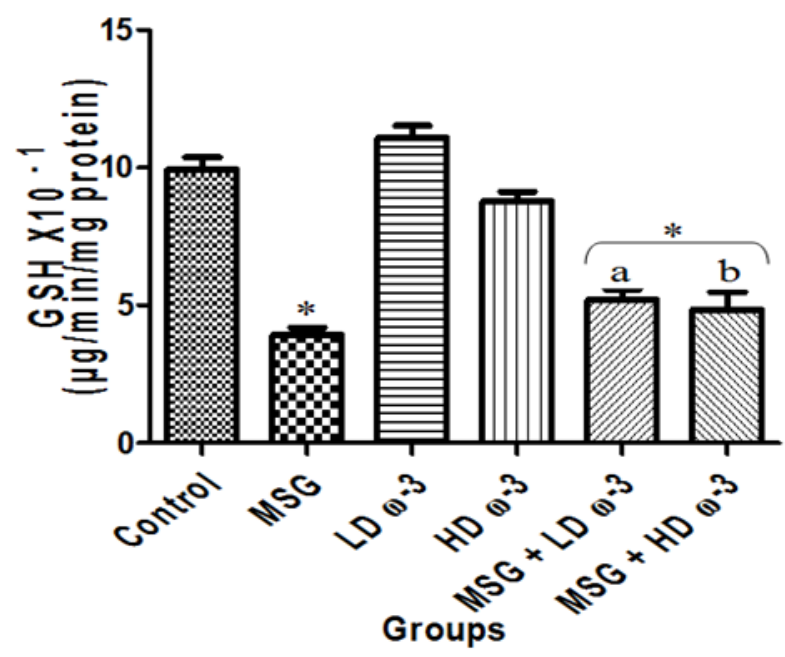

Graph 6. Effect of omega 3 fatty acids ( $\omega-3)$ on superoxide dismutase (SOD) in monosodium glutamate (MSG) treated Wistar rats. 


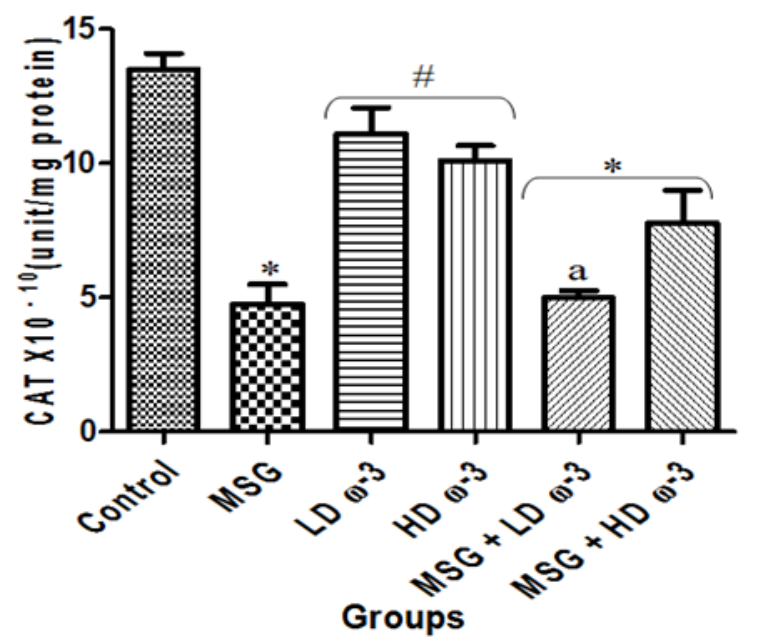

Graph 7. Effect of omega 3 fatty acids ( $\omega-3)$ on catalase (CAT) in monosodium glutamate (MSG) treated Wistar rats

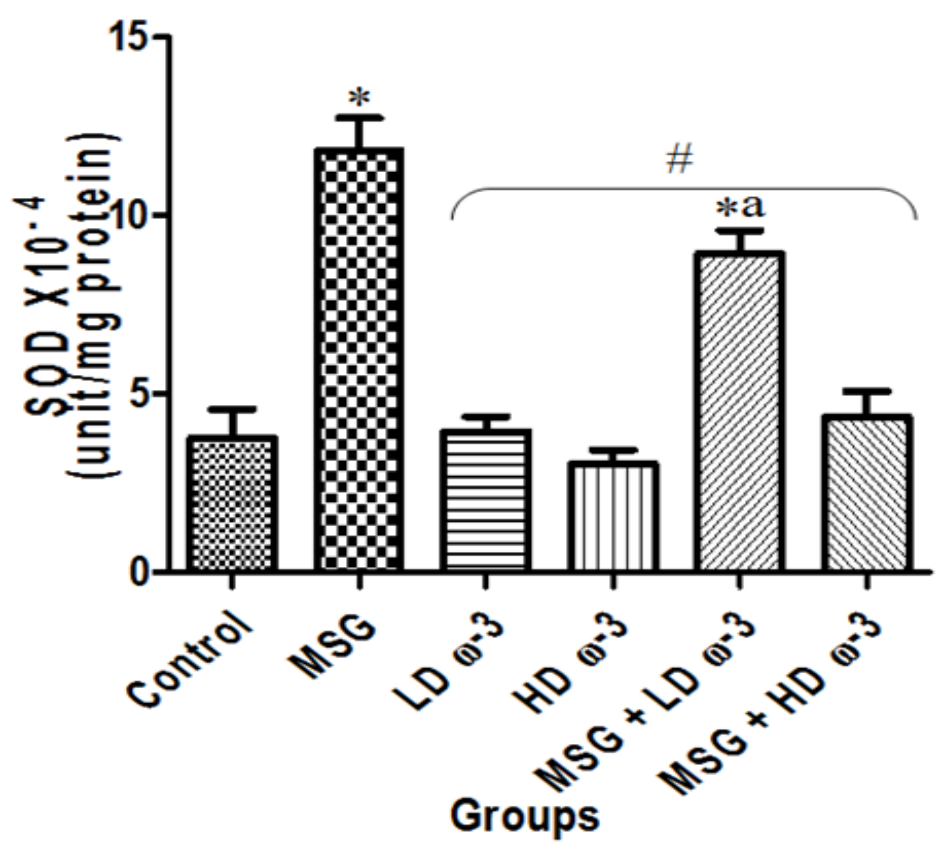

Graph 8. Effect of omega 3 fatty acids ( $\omega-3)$ on glutathione (GSH) in monosodium glutamate (MSG) treated male Wistar rats 
Table 1. Effect of omega 3 fatty acids on liver function enzymes in monosodium glutamate (MSG) - treated male Wistar rats

\begin{tabular}{|l|l|l|l|}
\hline $\begin{array}{l}\text { Groups } \\
\text { Parameters }\end{array}$ & $\begin{array}{l}\text { AST } \\
(\mathbf{U} / \mathbf{L})\end{array}$ & $\begin{array}{l}\text { ALT } \\
(\mathbf{U} / \mathbf{L})\end{array}$ & $\begin{array}{l}\text { ALP } \\
\text { (U/L) }\end{array}$ \\
\hline Control & $26.37 \pm 01.35$ & $16.91 \pm 01.71$ & $15.34 \pm 02.87$ \\
\hline MSG & $45.00 \pm 07.91^{* \#}$ & $44.54 \pm 01.71^{* \#}$ & $38.09 \pm 3.91^{* \#}$ \\
\hline LD $\omega-3$ & $26.66 \pm 04.13^{\#}$ & $18.99 \pm 05.81^{\#}$ & $20.33 \pm 02.78^{\#}$ \\
\hline HD $\omega-3$ & $27.95 \pm 02.89^{\#}$ & $20.28 \pm 02.34^{\#}$ & $17.89 \pm 02.65^{\#}$ \\
\hline MSG + LD $\omega-3$ & $45.19 \pm 10.47^{* a}$ & $34.88 \pm 03.23^{* \# a}$ & $31.53 \pm 5.83^{* \# a}$ \\
\hline MSG + HD $\omega-3$ & $34.20 \pm 07.92$ & $26.36 \pm 05.24^{* \# b}$ & $26.94 \pm 02.65^{* \# b}$ \\
\hline
\end{tabular}

Values were expressed as mean \pm SD, values with superscript * is significantly different at $p \leq 0.05$ when compared to control group; \# is significantly different at $p \leq 0.05$ when compared to MSG group; a indicate significant difference at $p \leq 0.05$ between LD $\omega-3$ and MSG + LD $\omega-3$; ${ }^{b}$ indicate significant difference at $p \leq 0.05$ between HD $\omega-3$ and MSG + HD $\omega-3$.

NB: AST - aspartate aminotransferase; ALT - aspartate aminotransferase; ALP alkaline phosphatase;

Table 2. Effect of omega 3 fatty acids on liver function Markers in monosodium glutamate (MSG) - treated Wistar rats

\begin{tabular}{lllll}
\hline $\begin{array}{l}\text { Groups/ } \\
\text { Parameters }\end{array}$ & $\begin{array}{l}\text { TB } \\
(\boldsymbol{\mu m o l} / \mathbf{L})\end{array}$ & $\begin{array}{l}\text { DB } \\
(\boldsymbol{\mu m o l} / \mathbf{L})\end{array}$ & $\begin{array}{l}\text { ALB } \\
(\mathbf{g} / \mathbf{d L})\end{array}$ & $\begin{array}{l}\text { TP } \\
\mathbf{( g / d L )}\end{array}$ \\
\hline Control & $6.25 \pm 01.24$ & $4.02 \pm 01.46$ & $5.37 \pm 01.38$ & $13.53 \pm 02.34$ \\
MSG & $13.69 \pm 04.08^{*} \#$ & $5.02 \pm 01.34$ & $2.16 \pm 01.39^{*} \#$ & $5.27 \pm 02.15^{*} \#$ \\
LD $\omega-3$ & $8.79 \pm 01.00 \#$ & $5.98 \pm 01.78$ & $3.16 \pm 00.28^{*}$ & $7.73 \pm 02.24^{*}$ \\
HD $\omega-3$ & $7.61 \pm 01.96 \#$ & $4.97 \pm 02.12$ & $5.00 \pm 01.96 \#$ & $12.86 \pm 02.39 \#$ \\
MSG + LD $\omega-3$ & $7.56 \pm 01.37 \#$ & $3.96 \pm 01.40$ & $3.68 \pm 0.98$ & $7.60 \pm 01.74^{*}$ \\
MSG + HD $\omega-3$ & $8.89 \pm 01.97 \#$ & $5.64 \pm 01.00$ & $3.75 \pm 0.69$ & $5.59 \pm 02.53^{*} \mathrm{~b}$
\end{tabular}

Values were expressed as mean \pm SD, values with superscript $*$ is significantly different at $p \leq 0.05$ when compared to control group; \# is significantly different at $p \leq 0.05$ when compared to MSG group; a indicate significant difference at $p \leq 0.05$ between LD $\omega-3$ and MSG + LD $\omega-3$; b indicate significant difference at $p \leq 0.05$ between HD $\omega-3$ and MSG + HD $\omega-3$.

NB: TB - total bilirubin; DB - direct bilirubin; ALB - albumin; TP- total protein

\subsection{Effect of graded doses of omega 3 fatty acids on hepatic function markers in monosodium glutamate (MSG) treated Wistar rats}

As shown in table 1 and 2, when compared to the control, there were significant $(p \leq 0.05)$ increase in the activities of AST in MSG and MSG + LD $\omega-3$ groups; ALT and ALP in MSG, MSG + LD $\omega-3$ and MSG + HD $\omega-3$ groups; also, there was significant $(p \leq 0.05)$ rise in the plasma TB level in MSG group. Contrarily, there were significant $(p \leq 0.05)$ decrease in plasma levels of ALB in MSG and LD $\omega-3$ groups and in TP level in MSG, LD $\omega-3$, MSG + LD $\omega-3$, and MSG + HD $\omega-3$ groups when compared to the control.

Relative to MSG group, there were significant $(p \leq 0.05)$ decrease in the activities of AST in LD $\omega-3$ and HD $\omega-3$ groups; ALT, ALP and levels of TB in groups 3-6 (LD $\omega-3$, HD $\omega-3$, MSG + LD $\omega-3$, and MSG + HD $\omega-3$ ). Also observed were significant $(p \leq 0.05)$ increase in plasma levels of ALB and TP in HD $\omega$-3 group compared to MSG. Associated with these changes were significant ( $p \leq 0.05$ ) increases in the activities of AST, ALT and ALP in MSG + LD $\omega-3$ group, relative to LD $\omega-3$ group and also significant $(p \leq 0.05)$ elevations in the activities of ALT and ALP in MSG + HD $\omega-3$ group, compared to HD $\omega-3$ group. 


\section{Discussion}

In this study, induction of monosodium glutamate toxicity was associated with an imbalance in the radox potential, altered lipid metabolism, compromised hepatic functions and hepatocellular damage. However, a synergic administration of EPA and DHA interrupted and ameliorated these pathological changes in a dose dependent pattern.

The dyslipidemia observed in this study was characterized by an increase in concentration of LDL and TAG, although this increase was not accompanied with a significant rise in the level of total cholesterol, but significant reduction in HDL concentration compared to the control. An increase in concentrations of TAG and LDL is a characteristics of cardiovascular disorder [27]. There are reports that MSG could intercept leptin processing and signaling leading to obesity [1], although Shi et al. [28] reported that prolong exposure to monosodium glutamate is not accompanied with weight gain. Amazingly, treatment with omega -3 fatty acid ameliorated these alterations in lipid metabolism, inferring that DHA and EPA are potent palliative agents for dyslipidemia. This intriguing properties of omega -3 on dyslipidemia observed in this study is in agreement with earlier reports $[14,18,29]$.

The observed increase in peroxidation of hepatic membrane lipids following exposure to MSG compared to the control, was a direct consequence of a compromised antioxidant system. Alteration in the radox potential due to generation of reactive oxygen species (ROS) and nitrogen species (RNS) as by-products from MSG metabolism and peroxidation of membrane lipids as well as inflammation of cells are the main complication associated excessive consumption of monosodium glutamate. Amazingly, a significant reduction in the level of MDA was seen in the low dose omega-3 fatty acids treated group relative to the MSG group indicating possible reversal of damage to hepatic membrane lipids. This finding concur with the report of Attia and Nasr [30], they reported that omega-3 fatty acid administration decreased membrane lipid peroxidation in the hepatocytes via reduction in MDA level in paraquat intoxicated rats.

In healthy conditions, SOD maintains the balance between the rate of $\mathrm{H}_{2} \mathrm{O}_{2}$ formation via dismutation of $\mathrm{O}_{-2}$ while the rate of removal of $\mathrm{H}_{2} \mathrm{O}_{2}$ is maintained by CAT and glutathione peroxidase. Hence, alteration or obstruction of this metabolic process will affect the activities of these enzymes involved. However, this study revealed non-significant changes in the activities of CAT and reduced glutathione concentrations when compared to MSG group. Though there were significant increase in the activities of SOD which could be due to endogenous mechanism of the physiological system to restore the ant-oxidative potential in the hepatic tissue. This finding is at variance with the report of earlier studies. Abdou and Hassan [31] reported significant increase in the level of GSH and activities of SOD and CAT in lead poisoned rats after treatment with omega-3 fatty acid. Also, Firat et al. [13] reported that omega -3 fatty acid supplementation corrected alterations associated with tissue levels of oxidant and antioxidant during regeneration. This study also revealed that administration of omega-3 fatty acid in the absence of MSG induction did not alter the oxidative stress profile but rather strengthen the antioxidant system. Hence, the ameliorative role of omega- 3 fatty acid on MSG induced toxicity cannot be denied.

The observed increase in AST and ALT in the MSG group relative to the control group is a consequence of hepatic damage to the cytoplasmic and mitochondria membranes. This was accompanied with hepatobiliary obstruction marked by the increase in ALP activities and TB levels. Interestingly, MSG deteriorating effect has been established in ovaries and testes of adult wistar rats [32]. Awesomely, relative to the MSG group, omega -3 fatty acid treatment significantly reduced the activities of ALP, ALT and AST, while increasing the rate of synthesis of albumin and other proteins, although this was not accompanied with significant changes in the conjugative functions of the liver. This outcome is contrarily, to previous report, that omega- 3 fatty acid treatment is not associated with significant changes in serum AST, ALT and ALP activities [30]. The hepatoprotective potentials of omega-3 fatty acid discovered in this study agrees with the report of Abdou and Hassan [31], they confirm the attenuation properties of omega-3 fatty acid on serum activities of AST, ALT ALP and restoration of serum proteins and albumin levels.

\section{Conclusion}

Treatment with LD and HD $\omega-3$ had no deleterious or adverse effect on the liver. Omega -3 intake strengthened the hepatic cells, hence LD $\omega-3$ are potent ameliorative supplement which can be administered after pre-exposure to MSG. 


\section{Compliance with ethical standards}

\section{Disclosure of conflict of interest}

The authors declare no conflicts of interest.

\section{Statement of ethical standards}

This study was carried out according to the guidelines stated in the directive 2010/63/EU as amended by Regulation (EU) 2019/1010 for the protection of animals used for scientific purposes.

\section{References}

[1] Bera TK, Kar SK, Yadav PK, Mukherjee P, Yadav S. Effects of monosodium glutamate on health. A systemic review. World Journal of Pharmaceutical Sciences. 2017; 5(5): 139-144.

[2] Elatrash MA, El-Haleim SZ. Protective roles of Ginko biloba on monosodium glutamate induced liver and kidney toxicity in rats. Research Journal of Pharmaceutical, Biological and Chemical Sciences. 2015; 6(1): 1433-1441.

[3] Newsholme P, Lima MM, Procopio J, et al. Glutamine and glutamate as vital metabolites. Brazilian Journal of Medical and Biological Research. 2003; 36(2): 153-163.

[4] Ogbuagu CE, Nweke IN, Unekwe PC. Organ Toxicity of monosodium glutamate in adult albino Wister rats. Journal of Medical Investigations and Practice. 2015; 10(1): 1-10.

[5] Lopez- Miranda V, Soto-Montenegro ML, Uranga-ocio JA, Vera G, Herradon E, Gonzalez C, Blas C, Lopez-Perez AE, Desco M, Abalo R. Effects of Chronic dietary exposure to monosodium glutamate on feeding behavior adiposity, gastrointestinal motility, and cardiovascular function in healthy adult rats. Neurogastroenterology and Motility. 2015; $27: 1559-1570$.

[6] Insawang T, Selmi C, Chaon U, Pethlert S, Yongvanit P, Areejitranusorn P, Boonsiri P, Khampitak T, Tangrassameeprasert R, Pinitsoontorn C, Prasongwattana V, Gershwin ME and Hammock BD. Monosodium glutamate intake is associated with the prevalence of metabolic syndrome in rural Thai population. Nutrition Metabolism (Lond.). 2012; 9(1): 50.

[7] Bautista RJH, Mahmoud AM, Konigsberg M and Guerreno NLD. Obesity: Pathophysiology, monosodium glutamate induced model and anti-obesity Medicinal plants. Biomedicine Pharmacotherapy. 2019; 111: 503-516.

[8] Kazmi Z, Fatima I, Perveen S and Malik SS. Monosodium glutamate: Review on Clinical reports. International Journal of food properties. 2017; 20(52): S1807-S1815.

[9] Siscovick DS, Barringer TA, Frett AM, Wu JHY, Lichtenstein AH, Costello RB, Kris-Etherton PM, Jacobson MD, Engler MB, Alger HM, Appel LJ and Mozaffarian D. Omega- 3 Ployunsaturated fatty acid (Fish oil) supplementation and prevention of clinical cardiovascular disease. Journal Circulation. 2017; 135: e867-e884.

[10] Durmus M. Fish oil for human health: Omega-3- fatty acid profiles of marine seafood species. Food Science and Technology. 2018; 1-8.

[11] Leigh-Firbank EC, Minihane AM, Leake DS, Wright JW, Murphy MC, Griffin BA, Willams CM. Eicosapentanoic acid and docosahexanoic, acid form fish oils: differential associations with Lipid Responses. British Journal of Nutrition. 2002; 87: 435-445.

[12] Al-Attar AM, AL-Rethear HA. Chemoprotective effect of Omega- 3-fatty acids on thioacetamide induced hepatic fibrosis in male rats. Saudi Journal of Biological Science. 2017; 24(4): 956-965.

[13] Firat O, Makay O, Yeniary L, Gokce G, Yenisey C and Coker A. Omega-3 fatty acids exhibit oxidative stress in a rat model of liver regeneration. Annals of Surgical Treatment Research. 2017; 93(1): 1-10.

[14] Swanson D, Block R, Mousa SA. Omega-3 fatty acids EPA and DHA: Health Benefits throughout life, Systemic review. Advanced Nutrition. 2012; 3: 1-7.

[15] Mateous HT, Lewondowski PA, Vaughon V, Su XQ. Health impacts of eicosapentanoic acid and docosahexanoic acid. CAB Reviews. 2013; 8(7): 1-12. 
[16] Rendeiro C, Sheriff A, Bhattacharya TA, Gogola JV, Baxter JH, Chen H, Helferich WG, Roy EJ, Rhodes JS. Long lasting impairment in adult neurogenesis, spatial learning and memory from a standard chemotherapy regimen used to treat breast cancer. Behavioral Brain Research. 2016; 315: 10-22.

[17] Kasavalu L, Vasudevan B, Raghu B, Browning E, Dawson D, Novak JM, Correll MC, Steffen MJ, Bhattacharya A, Fernandes G, Ebersole JL. Omega-3 fatty acid effect on aveolar bone loss in rats. Journal Dent Research. 2006; 85(7): 648-652.

[18] Brown AL, Zhu X, Rong S, Shewale S, Seo J, Boudyguina E, Gebre AK, Alexandra-Miller MA, Parks JS. Omega-3 fatty acids ameliorate atherosclerosis by favorably altering monocyte subsets and limiting monocyte recruitments to aortic lesions. Arteriosclerosis, Thrombosis and Vascular Biology. 2012; 32: 2122-2130.

[19] Balk EM, Lichtenstein AH. Omega-3 fatty acids and cardiovascular diseases: summary of the 2016 Agency of health care research and quality evidence review. A systematic review. Nutrients. 2017; 9(865): 1-13.

[20] Vandongen R, Mori TA, Burke V, Beilin LJ, Moris J, Ritche J. Effects on Blood pressure of $\omega 3$ fats in subjects at increased risk of cardiovascular disease. Hypertension. 1993; 22 (3): 372 - 379.

[21] Reagan-Shaw S, Nihal M, Ahmad N. Dose translation from animal to human studies revisited. FASEB Journal. 2008; 22(3): 659-661.

[22] Sedlak J, Lindsay RH. Estimation of total, protein bound and non-protein sulfhydryl groups in tissue with Ellman's reagent. Analytical Biochemistry. 1968; 25: 192.

[23] Hunter FE, Gebicki JM, Hoffstein PE, Weinstein J, Scott A. Swelling and lysis of rat liver mitochondria induced by ferrous ions. Journal of Biology and Chemistry. 1963; 238: 828-835.

[24] Gutteridge JMC, Wilkins C. Copper-dependent hydroxyl radical damage to ascorbic acid. Formation of a thiobarbituric acid reactive products. FEBS Letters. 1982; 137: 327-340.

[25] Beers RF, Sizer IW. A spectrophotometric method for measuring the breakdown of hydrogen peroxide by catalase. Journal of Biological Chemistry. 1952; 195, 130-140.

[26] Mistra HP, Fridovich I. The role of superoxide anion in the auto-oxidation of epinephrine and a single assay for superoxide dismutase. Journal of Biological Chemistry. 1972; 247: 3170-3175.

[27] Burtis CA, Ashwood ER. Fundamentals of clinical chemistry, fifth edition,W.B. Saunders company, New York. 1953.

[28] Shi Z, Luscombe-merch, ND, Wittert GA, Yuan B, Dai Y, Pan X, Taylor AW. Monosodium glutamate is not associated with obesity or a greater prevalence of weight gain over 5years: findings from Jiangsu nutrition study of Chinese adults. British Journal of Nutrition. 2010; 1004(3): 457-463.

[29] Back N. Omega -3 fatty acids in atherosclerosis and coronary artery disease. Future Science. 2017; 3(4): 20362056.

[30] Attia AM, Nasr HM. Evaluation of protective effect of omega-3 fatty acids and selenium on paraquat intoxicated rats. Slovak Journal of Animal Science. 2009; 42(4):180-187.

[31] Abdou HM, Hassan MA. Protective role of Omega -3 polyunsaturated fatty acid against Lead acetate induced toxicity in liver and kidney of female rats. Biomed Research International. 2014; 35857.

[32] Eweka AO, Om-Iniabohs FAE. Histological study of the effect of monosodium glutamate on the ovaries of adult wistar rats. Annals of Medical and Health Science Research. 2011; 1(1): 37-44. 